\title{
Harmonic Source Location in the Distribution Grid Using Time- Synchronized Measurements
}

\author{
S. Babaev ${ }^{1}$, V. Ćuk ${ }^{1}$, J.F.G. Cobben ${ }^{1}$ and H.E. van den Brom ${ }^{2}$ \\ ${ }^{1}$ Department of Electrical Engineering \\ Eindhoven University of Technology (TU/e) \\ 5600MB Eindhoven (Netherlands) \\ E-mail: S.Babaev@tue.nl \\ ${ }^{2} \mathrm{VSL}$ \\ 2629JA Delft (Netherlands) \\ E-mail: hvdbrom@vsl.nl
}

\begin{abstract}
A method for locating harmonic producing source in the low-voltage distribution network is presented. The method aims to employ natural load variability of the existing non-linear installations. By performing multi-point time-synchronized acquisition of the voltage and current waveforms and analysing spectral characteristics of the signals, harmonic impedance of the cable section associated with non-linear load is estimated. A certain rejection algorithm is applied in order to discard data sets with unreliable information. To validate the method, a field test was established in the low-voltage grid. The results demonstrate capability of the proposed technique to accurately estimate harmonic impedances and successfully identify location of harmonic current source in the grid.
\end{abstract}

\section{Key words}

Power quality, Power system harmonics, Impedance measurement, Power system measurements.

\section{Introduction}

The phenomenon of the harmonic propagation draws more attention during the last decade. It is because of that phenomenon the harmonic voltages at the different points of the power system do not necessary match the connecting node of the dominant harmonic producing source. The underlying reason is the impedances of the system which govern the level of propagation of harmonic disturbances. Therefore, by accurately estimating frequency properties of the system, it is feasible not only to evaluate contributions to harmonic pollution of load and utility but also to track physically the dominant harmonic producing source.

A comprehensive summary of the existing methods for calculating harmonic impedances has been presented in [1], where authors pointed out two different approaches. These methods aimed to employ either natural load variations in the power system (on-line non-invasive methods) or, in contrast, direct injection of harmonic currents (on-line invasive methods).

Described techniques has been further developed and applied in [2],[3],[4] and [5].

Furthermore, in studies [6] and [7] authors have introduced methods for determining harmonic current contributions from various sources. The latter was performed using sample-by-sample time-synchronized voltage and current measurements.

Authors of [8] demonstrated another approach for determining harmonic impedance of the system by employing data from several Phasor Measurement Units (PMUs) which were reporting time-synchronized voltage and current measurements.

Despite the time varying nature of harmonic impedances, few studies report identification of harmonic sources through application of methods involving precise timing procedures.

A major challenge is in setting up an appropriate experiment in the distribution grid, which engages specific measurement equipment featuring time-synchronization.

This paper presents a method for locating harmonic producing source in the distribution network. The established experiment aims to utilize multi-point timesynchronized measurements for accurate estimation of harmonic impedances.

In the following section the proposed approach is described. Section 3 provides detailed information about field experiment and achieved results. After brief discussion in section 4, this paper is followed by the conclusion.

\section{Proposed Method}

\section{A. Harmonic Propagation}

The proposed method aims to utilize natural load variability, therefore it is referred to the non-invasive 
methods. Thus, current and voltage harmonics variations are used for harmonic impedance estimation. The underlying principle is the harmonic propagation phenomenon, which causes voltage harmonics to be produced in several points of the grid by flowing harmonic currents. Consequently, depending on the state and parameters of the network, the connecting point of the nonlinear load does not necessary match the node with highest harmonic voltage.

Given a small part of radial power system (Fig. 1), a set of the equations may be written:

$$
\begin{gathered}
V_{S 1}(f)=V_{e 1}(f)+Z_{A B} I_{L 1}(f)+Z_{B C} I_{e}(f) \\
V_{S 2}(f)=V_{e 2}(f)+Z_{A B} I_{L 2}(f)+Z_{B C} I_{e}(f)
\end{gathered}
$$

where $V_{S}(f)$ and $V_{e}(f)$ are complex voltage spectra values at the transformer terminals and end of the cable, respectively, $Z_{A B}(f)$ and $Z_{B C}(f)$ are harmonic impedances of the cable sections from power source to harmonic load and from load to the end of the cable, respectively, and $I_{L}(f), I_{e}(f)$ are complex current spectra of feeder under the study and other non-linear loads presented in the system, respectively. The subscript 1 represents the state of the power system with dominating harmonic source being switched-off whereas subscript 2 stands for the moment of time with operating harmonic source.

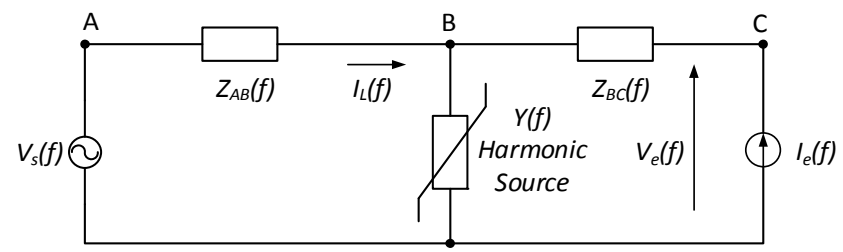

Fig. 1. Power System model for harmonic impedance estimation

Assuming that the change of the state is provoked mainly by the suspected load and implying other loads do not cause significant change in the system at this particular time, the subtraction of (1) and (2) gives the following result:

$$
\Delta V_{S}(f)-\Delta V_{e}(f)=Z_{A B}\left(I_{L 1}(f)-I_{L 2}(f)\right)
$$

Accordingly, the harmonic impedance of the cable section is calculated as:

$$
Z_{A B}(f)=\frac{\Delta V_{S}(f)-\Delta V_{e}(f)}{I_{L 1}(f)-I_{L 2}(f)}
$$

As a result, having estimated the impedance of the cable section at frequencies associated with the operation of dominant harmonic source, the location of this source can be determined.

\section{B. Synchronized Measurements}

The substantial impact on the performance of described method is governed by the measurement system. As may be inferred from the equations presented above, the method is worthwhile only when measurements are available in at least two points of the power grid. Furthermore, in order to estimate impedance accurately it is proposed to synchronize current and voltage measurements obtained from different acquisition systems using deterministic time source.

\section{Application to Field Measurements}

The proposed method is verified by setting up an experiment in a low-voltage grid in the Netherlands. The equivalent impedance of the cable section is estimated and location of the dominating harmonic producing load is identified.

\section{A. Experimental Setup}

In order to gain a certain level of control over the experiment an external programmable load bank comprising set of non-linear appliances with a total power of $11.4 \mathrm{~kW}$ was connected to one of the low-voltage feeders.

Voltage measurements took place at the low-voltage terminals of the $10 / 04 \mathrm{kV}$ transformer supplying various residential as well as commercial buildings. Secondly, another voltage measurement device was placed in the cabinet at the end of the cable to which external load was connected. Current waveforms were recorded at the beginning of the same feeder. The rated power of the transformer and short-circuit voltage are $400 \mathrm{kVA}$ and $4 \%$, respectively. The simplified equivalent diagram of the network is depicted in Fig. 2.

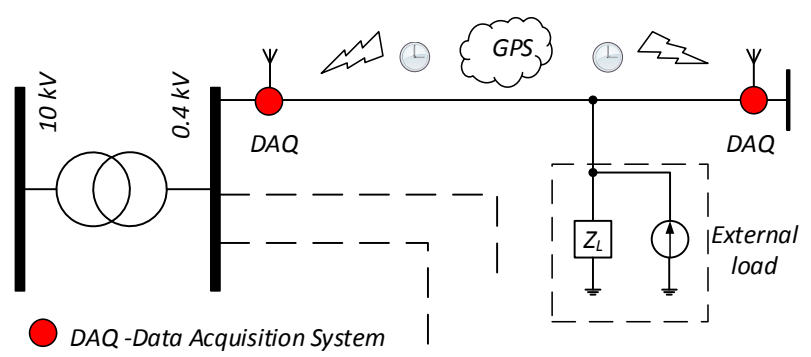

Fig. 2. Experimental setup

The experiment was performed with provision of National Instruments (NI) data acquisition system built on chassis cRIO-9068 and cRIO-9038, which featured NI9225 and NI9234 measurement cards with predefined sampling frequency of $5 \mathrm{kHz} / \mathrm{s}$. The current waveforms were recorded with current clamp-on sensors Fluke i200s with basic accuracy of $3 \%$ for $50 \mathrm{~Hz}$. Additional error of $3 \%$ applies for higher frequencies. Further, GPS modules with antennas were installed to each of the chassis. The latter provided synchronous start of the measurements triggered by arriving Pulse-per-Second (PPS) from GPS.

\section{B. Conditions of Experiment and Initial Data}

An external harmonic producing load exhibited periodic switching driven by PLC controller, specifically at each sub-cycle of switching sequence the power of the nonlinear loads varied. Further, the duration of the complete switching cycle was approximately $24 \mathrm{~min}$. Fig. 3 presents 1-cycle RMS values of voltages and currents acquired synchronously under this experiment. For the sake of 
simplicity, only measurements of phase A are included in this paper.
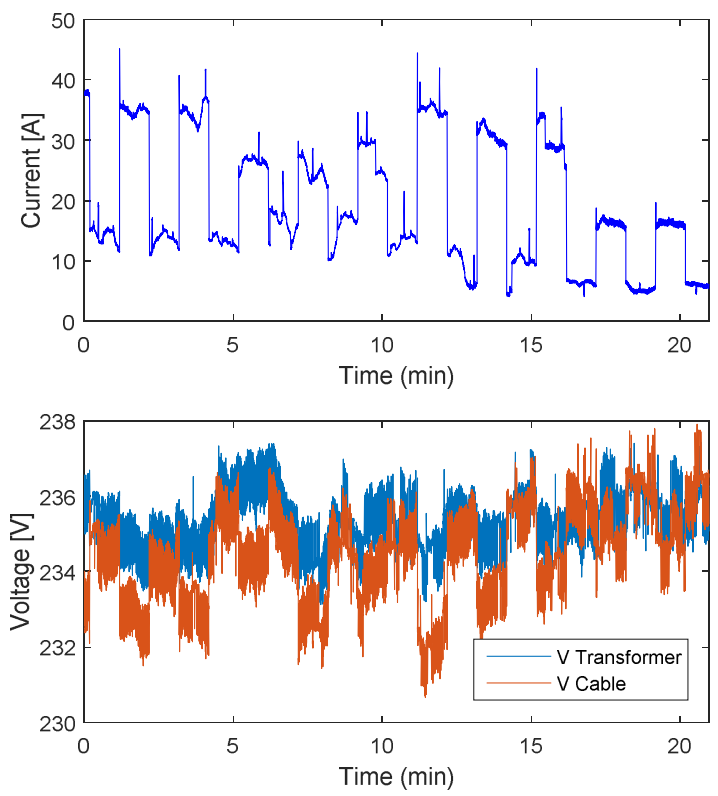

Fig. 3. Initial RMS of current and voltage

From the presented figures different switching moments of the load can be seen, resulting in various levels of load currents and associated voltage drops.

\section{Estimation of Impedances}

The accuracy of impedance estimation in non-invasive experiments is affected to a great extent by presence of background voltage harmonics, weak excitation and variation of the harmonic impedance itself over the course of the time. Therefore, several data selections might contain redundant information which leads to erroneous results. Thus, a selection algorithm is applied in order to reject unreliable segments of the data.

Fig. 4 illustrates the results of Fast Fourier Transform (FFT) analysis of the current waveform, which corresponds to the time instance when external load was switched on and off between 2 and $5 \mathrm{~min}$. This figure comprises FFT of the current drawn by all the loads connected to this particular feeder. It can be observable that 7 th harmonic component is prominent at a relatively high level during operation of the load (red bar) whereas upon steady-state periods (blue and dark-blue bars) the FFT-spectra indicates presence of the currents due to the background harmonic voltage or other harmonics loads. The notion "steady-state" reflects the instances of time when load under study was switched off while all other possible customers remained active.

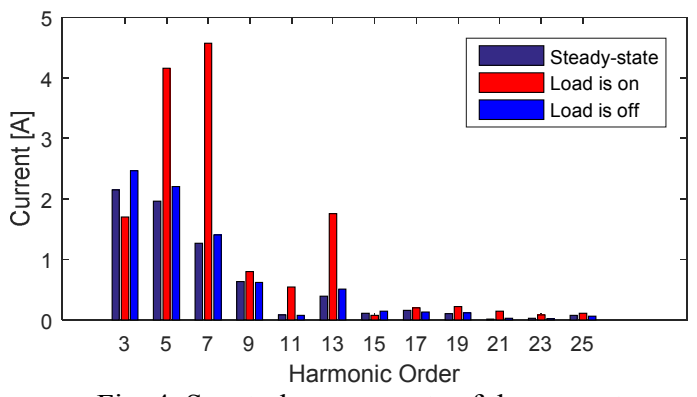

Fig. 4. Spectral components of the current
Fig. 5 presents the magnitude spectrum of voltages obtained synchronously at the same instant between 2 and $5 \mathrm{~min}$. It is evident that strong correlation exists between increased emission and produced harmonic voltage. It is worth noting that, although, the level of excitation at 250 $\mathrm{Hz}$ component is comparably high, the estimation of impedance at this frequency is unreliable due to the large harmonic background voltage and its drastic variation during execution of switching sequence of the load. Interestingly, the reduction of the $3^{\text {rd }}$ order harmonic current leads to the increase of the corresponding voltage at the end of the feeder. This is possibly due to the harmonic cancellation effect or sensitivity issues.

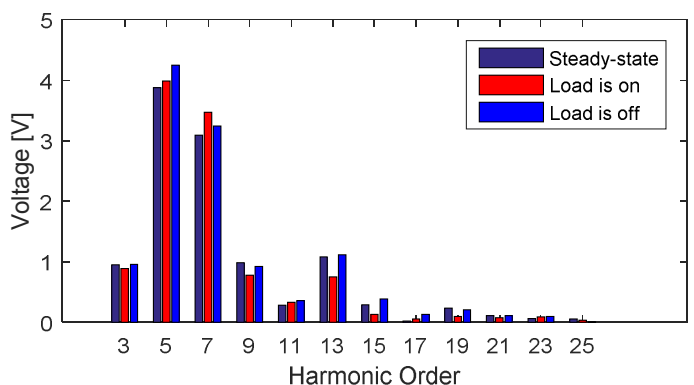

(a)

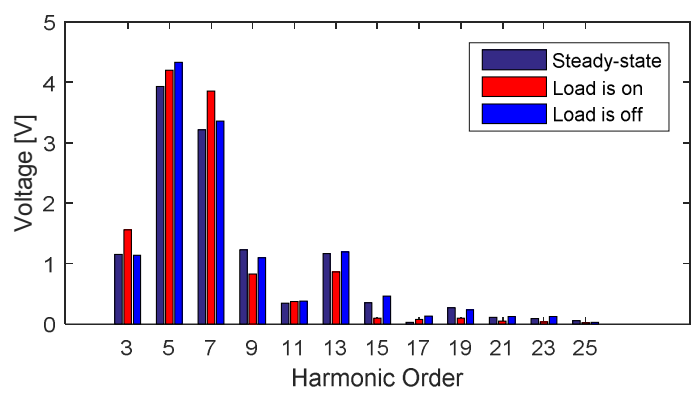

(b)

Fig. 5. (a) Output of FFT-analysis of voltage at the transformer terminals. (b) Spectral components of the voltage at the end of the cable

Table I comprises four different data selections and demonstrates initial steady-state voltages and currents and their relational increase during operation of the load. Based on the conclusions presented above, the 7th harmonic component is chosen for the impedance estimation. Moreover, in order to validate the obtained results, the fundamental component is also included in studies. These data selections correspond to the four general cases:

1) high harmonic excitation and initial high loading level;

2) high harmonic excitation and initial low loading level;

3) low harmonic excitation and initial high loading level;

4) low harmonic excitation and initial low loading level;

Referring to the section 1, the harmonic impedances of the cable section are computed for every case out of which results originated from first two cases demonstrate consistent impedance values whereas output from the 
Table I. - Initial steady-state values and relative change

\begin{tabular}{|c|c|c|c|c|c|c|c|}
\hline \multirow{2}{*}{ Case } & $\mathrm{h}, \#$ & Current, $\mathrm{A}$ & $\mathrm{V}_{\text {trans, }} \mathrm{V}$ & $\mathrm{V}_{\text {cable, }} \mathrm{V}$ & $\Delta \mathrm{l}, \%$ & $\Delta \mathrm{V}_{\text {trans, } \%}$ & $\Delta \mathrm{V}_{\text {cable, } \%}$ \\
\hline \multirow{2}{*}{1} & 1 & 11.45 & 235.83 & 235.25 & 195 & -0.21 & -0.77 \\
\cline { 2 - 8 } & 7 & 1.27 & 3.09 & 3.21 & 259 & 12.29 & 19.93 \\
\hline \multirow{2}{*}{2} & 1 & 5.34 & 235.73 & 234.27 & 448 & -0.2 & -0.27 \\
\cline { 2 - 8 } & 7 & 1.48 & 3.38 & 2.92 & 197.72 & 7.97 & 33.2 \\
\hline \multirow{2}{*}{3} & 1 & 11.05 & 235.98 & 235.42 & 106.75 & -0.09 & -0.67 \\
\cline { 2 - 8 } & 7 & 1.3 & 3.27 & 3.4 & 133.07 & 3.68 & 8.93 \\
\hline \multirow{2}{*}{4} & 1 & 5.18 & 235.67 & 234.77 & 168.81 & -0.097 & -0.17 \\
\cline { 2 - 8 } & 7 & 1.38 & 3.34 & 2.85 & 120.77 & 3.91 & 34.57 \\
\hline
\end{tabular}

realizations with low harmonic excitation is regarded rather erroneous. Furthermore, a histogram of the impedance values from successful sets of data allows to approximate a base value to be utilized for calculation of the distance. Results are collected in Table II for fundamental and 7th harmonic component. The distances up to splitting point are computed based on $95 \mathrm{~mm}^{2}$ aluminium cable and compared to actual distance (data provided by Distribution Network Operator).

Table II. - Calculated impedances and distances to harmonic source

\begin{tabular}{|c|c|c|c|c|}
\hline \multirow{2}{*}{$\mathrm{h}, \#$} & \multirow{2}{*}{$|\mathrm{Z}|, \mathrm{Ohm}$} & \multicolumn{2}{|c|}{ Distance, $\mathrm{km}$} & \multirow{2}{*}{ Relative error, \% } \\
\cline { 3 - 4 } & & Computed & Actual & \\
\hline 1 & 0.0563 & 0.1562 & \multirow{2}{*}{0.129} & 17.41 \\
\hline 7 & 0.0727 & 0.1176 & & 9.69 \\
\hline
\end{tabular}

D. Estimation of harmonic impedance as a frequencyresponse of the system

Typically, when the system loading level is low and harmonic excitation is weak in the presence of background harmonics then the measurements to be performed in such a system are prone to noise and impaired sensitivity. As it is shown in [9] in order to increase signal-to-noise ratio (SNR), the impedance might be represented as the frequency response of linear time-invariant (LTI) system. The input to that system is represented as a stationary random process and the output is averaged. By estimating power spectral density (PSD) of the input and cross power spectral density of the input and output, the frequency response of the LTI system is computed as follows:

$$
Z(f)=\frac{P_{x y}(f)}{P_{x x}(f)}
$$

In order to obtain an estimate of the power spectrum, a method of periodograms is applied, where periodogram is a convolution of a true power spectral density and FFT of autocorrelated window sequence [10]. Further, the accuracy of the estimate by means of periodogram is strongly dependent on applied window length and number of DFT points to be used for calculation. In order to be able to obtain a smooth spectrum considering trade between variance and the resolution the described procedure was further developed to the method of averaged periodograms by Welch. The signal in such a method is broken down to a number of segments and thereafter the estimates of the periodogram are averaged.

Fig. 6a shows an example of two voltage waveforms corresponding to the transformer terminals and the end of the cable and recorded during transition of the external load from on to the off state whereas Fig. $6 \mathrm{~b}$ represents harmonic impedances computed with Welch's averaged periodogram.

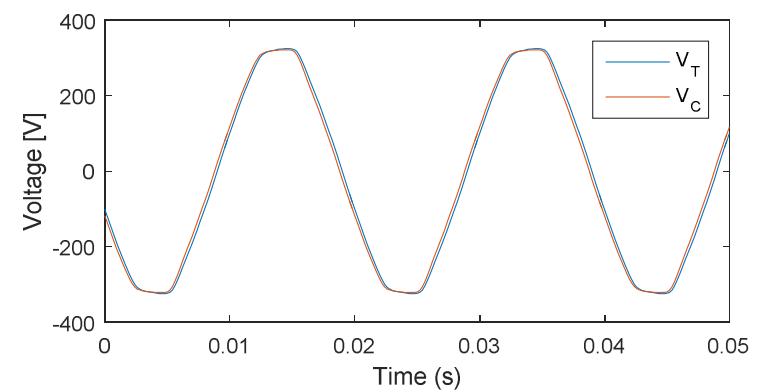

(a)

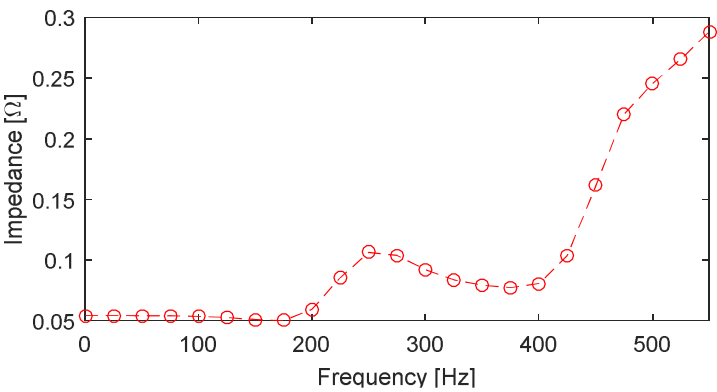

(b)

Fig. 6. (a) Synchronized voltage waveforms. (b) Estimated impedances

Table III shows the comparison between results obtained by means of Welch's averaged periodogram and classic DFT method for that particular state of the power network.

Table III. - Comparison of impedance values obtained with Welch's averaged periodogram and classic DFT

\begin{tabular}{|c|c|c|c|c|c|}
\hline \multirow{2}{*}{ Method } & $\begin{array}{c}\mathrm{h}, \\
\#\end{array}$ & $\begin{array}{c}|\mathrm{Z}|, \\
\text { Ohm }\end{array}$ & $\mathrm{S}, \mathrm{km}$ & $\begin{array}{c}\text { Actual } \\
\mathrm{S}, \mathrm{km}\end{array}$ & $\begin{array}{c}\text { Error, } \\
\%\end{array}$ \\
\hline \multirow{2}{*}{ Welch } & 1 & 0.0541 & 0.1501 & & 14.06 \\
\cline { 2 - 4 } & 7 & 0.0792 & 0.1281 & \multirow{2}{*}{0.129} & 0.7 \\
\hline \multirow{2}{*}{ DFT } & 1 & 0.0539 & 0.1496 & & 13.77 \\
\cline { 2 - 3 } \cline { 6 - 6 } & 7 & 0.0236 & 0.0382 & & $>100$ \\
\hline
\end{tabular}




\section{Discussions}

Natural load variability generally provides weak harmonic excitation levels. Presented results, however, demonstrate that even in that case, given that a certain rejection algorithm is applied, it is though feasible to accurately determine utility and cable impedance values and track the source of the harmonics in the system. As it may be deduced from results, in certain cases the change of the fundamental component of the voltage is negligible, typically at the uncertainty range of the measurement chain, therefore providing false estimation of impedances. It is worth noting, that none of the cases involving fundamental spectral component gave relative error less than $13 \%$.

Nevertheless, the sensitivity of measurements can be significantly better if higher spectral components are taken into consideration for calculation. Thus, it is important to properly characterize measurement instrumentation and choose devices with certain accuracy levels which to be adapted for the specific purpose of the experiment.

Furthermore, deploying multi-point measurements and having an aim to determine harmonic impedances, it is necessary to provide time synchronization with the highest possible accuracy, preferably not worse than $200 \mathrm{~ns}$. It is dictated by the fact that parameters being recorded at not truly synchronized time instance at different points of the network will likely be affected by other events evolving in the system.

Moreover, one of the possible ways to reduce system noise is the application of the Welch's averaged periodogram, which proved to be promising based on the calculated relative error of $0.7 \%$.

None of the methods discussed above either solves the problem of the spectral leakage or picket fence. Likewise, it is recommended to have samples taken for FFT analysis synchronized to power frequency, which presents challenge for some of the available high-precision measurement systems. It is therefore might be beneficial to analyze multipoint synchronized measurements by means of other spectral processing methods, namely Short-time Fourier transform (STFT) or Wavelets transform. This is left for the future research work.

\section{Conclusions}

This paper presents a method for identifying the location of the dominating harmonic current source in the low-voltage power system. A case study involving one feeder of the real power system is discussed. An additional controllable nonlinear load was connected at the random point along that particular cable. Based on the harmonic impedances, which determine the propagation level of harmonics, a non-linear load can be physically tracked in the power system. This study might be further expanded to more complicated cases involving several feeders and connection points. Based on the input from distribution system operator, i.e. types of the cables and associated lengths the so called impedancefitting procedure might be applied to determine the location of harmonic load.

Considering a natural variability of the loads and continuous change of the power system state over the course of the time, the proposed method gives satisfactory results only when multi-point measurements of voltages and currents are applied. Furthermore, these data acquisition devices need to be synchronized to the precise timing source. In this paper it is proposed to use GPS for timesynchronization. Among the advantages of the method are utilization of the natural load variability and its ability to track accurately dynamic events due to the synchronization with the common clock. This mainly applies to the cases when harmonic current injection of the particular non-linear load is at relatively high level. Improving sensitivity of the method, quality of the time synchronization and dealing with drawbacks of DFT are topics for the future research study.

\section{Acknowledgement}

This work has received funding from the European Union's Horizon 2020 research and innovation programme under the Marie Skłodowska-Curie grant agreement №676042.

\section{References}

[1] A. Robert and T. Deflandre, "Guide for assessing the network harmonic impedance," in Proc. 14th Int. Conf. Exhib. on Electricity and Distribution (CIRED), Stockholm, 1997.

[2] M. Sumner, B. Palethorpe, D. W. P. Thomas, P. Zanchetta, and M. C. Di Piazza, "A technique for power supply harmonic impedance estimation using a controlled voltage disturbance," IEEE Trans. Power Electron., vol. 17, no. 2, pp. 207-215, 2002.

[3] M. Ciobotaru, R. Teodorescu, and F. Blaabjerg, "On-line grid impedance estimation based on harmonic injection for grid-connected PV inverter," IEEE Int. Symp. Ind. Electron., no. i, pp. 2437-2442, 2007.

[4] F. Karimzadeh, S. Esmaeili, and S. H. Hosseinian, "A Novel Method for Noninvasive Estimation of Utility Harmonic Impedance Based on Complex Independent Component Analysis," Power Deliv. IEEE Trans., vol. 30, no. 4, pp. 1843-1852, 2015.

[5] R. Langella and A. Testa, "A new method for statistical assessment of the system harmonic impedance and of the background voltage distortion," 2006 9th Int. Conf. Probabilistic Methods Appl. to Power Syst. PMAPS, no. 2, pp. 9-15, 2006.

[6] P. S. Chandramohanan Nair and F. M. Fernandez, "Method for separation of customer and utility contributions of harmonics at point of common coupling," IET Gener. Transm. Distrib., vol. 7, no. 4, pp. 374-381, 2013.

[7] O. Unsar, O. Salor, I. Cadirci, and M. Ermis, "Identification of harmonic current contributions of iron and steel plants based on time-synchronized field measurements - Part I: At PCC," IEEE Trans. Ind. Appl., vol. 50, no. 6, pp. 4336 $4347,2014$.

[8] V. Cuk et al., "Measurement of the harmonic impedance of the aggregated distribution network," 2016 17th Int. Conf. Harmon. Qual. Power, pp. 739-744, 2016.

[9] D. Borkowski, A. Wetula, and A. Bien, "New method for noninvasive measurement of utility harmonic impedance," IEEE Power Energy Soc. Gen. Meet., vol. 1, no. 1, pp. 2-9, 2012.

[10] A.V. Oppenheim, R.W. Schafer, and J.R. Buck, "DiscreteTime Signal Processing. Upper Saddle River, NJ, USA: Prentice Hall, 1999. 